International Mathematical Forum, Vol. 9, 2014, no. 5, 237 - 254

HIKARI Ltd, www.m-hikari.com

http://dx.doi.org/10.12988/imf.2014.37134

\title{
Applications of Slowly Changing Functions in the Estimation of Growth Properties of Composite Entire Functions on the Basis of their Maximum Terms and Maximum Moduli
}

\author{
Sanjib Kumar Datta \\ Department of Mathematics,University of Kalyani \\ Kalyani, Dist-Nadia,PIN- 741235, West Bengal, India
}

Tanmay Biswas

Rajbari, Rabindrapalli, R. N. Tagore Road

P.O.- Krishnagar, Dist-Nadia

PIN- 741101, West Bengal, India

Azizul Haque

Gobargara High Madrasah (H.S.)

P.O.+P.S.- Hariharpara, Dist-Murshidabad

PIN- 742166, West Bengal, India

Copyright (c) 2014 Sanjib Kumar Datta, Tanmay Biswas and Azizul Haque. This is an

open access article distributed under the Creative Commons Attribution License, which permits unrestricted use, distribution, and reproduction in any medium, provided the original work is properly cited.

\begin{abstract}
In the paper we prove some comparative growth properties of composite entire functions on the basis of their maximum terms and maximum moduli using generalised $L^{*}$-order and generalised $L^{*}$-lower order.
\end{abstract}

Mathematics Subject Classification: 30D35, 30D30 
Keywords: Entire function, maximum term, maximum modulus, composition, growth, slowly changing function, generalised $L^{*}$-order (generalised $L^{*}$-lower order)

\section{Introduction, Definitions and Notations.}

Let $\mathbb{C}$ be the set of all finite complex numbers and $f$ be an entire function defined in $\mathbb{C}$. The maximum term $\mu(r, f)$ of $f=\sum_{n=0}^{\infty} a_{n} z^{n}$ on $|z|=r$ is defined by $\mu(r, f)=\max _{n \geq 0}\left|a_{n}\right| r^{n}$ and the maximum modulus $M(r, f)$ of $f$ on $|z|=r$ is defined as $M(r, f)=\max _{|z|=r}|f(z)|$. We use the standard notations and definitions in the theory of entire functions which are available in [11]. In the sequel we use the following notation :

$$
\log ^{[k]} x=\log \left(\log ^{[k-1]} x\right) \text { for } k=1,2,3, \ldots \text { and } \log ^{[0]} x=x .
$$

To start our paper we just recall the following definitions :

Definition 1 The order $\rho_{f}$ and lower order $\lambda_{f}$ of an entire function $f$ are defined as

$$
\rho_{f}=\limsup _{r \rightarrow \infty} \frac{\log ^{[2]} M(r, f)}{\log r} \text { and } \lambda_{f}=\liminf _{r \rightarrow \infty} \frac{\log ^{[2]} M(r, f)}{\log r} .
$$

Extending this notion, Sato [6] defined the generalised order and generalised lower order of an entire function as follows :

Definition 2 [6]Let $m$ be an integer $\geq 2$. The generalised order $\rho_{f}^{[m]}$ and generalised lower order $\lambda_{f}^{[m]}$ of an entire function $f$ are defined by

$$
\rho_{f}^{[m]}=\limsup _{r \rightarrow \infty} \frac{\log ^{[m]} M(r, f)}{\log r} \text { and } \lambda_{f}^{[m]}=\liminf _{r \rightarrow \infty} \frac{\log ^{[m]} M(r, f)}{\log r}
$$

respectively.

For $m=2$, Definition 2 reduces to Definition 1 .

If $\rho_{f}<\infty$ then $f$ is of finite order. Also $\rho_{f}=0$ means that $f$ is of order zero. In this connection Datta and Biswas [2] gave the following definition :

Definition 3 [2]Let $f$ be an entire function of order zero. Then the quantities $\rho_{f}^{* *}$ and $\lambda_{f}^{* *}$ of $f$ are defined by:

$$
\rho_{f}^{* *}=\limsup _{r \rightarrow \infty} \frac{\log M(r, f)}{\log r} \text { and } \lambda_{f}^{* *}=\liminf _{r \rightarrow \infty} \frac{\log M(r, f)}{\log r} .
$$


Let $L \equiv L(r)$ be a positive continuous function increasing slowly i.e., $L(a r) \sim L(r)$ as $r \rightarrow \infty$ for every positive constant $a$. Singh and Barker [7] defined it in the following way:

Definition 4 [7]A positive continuous function $L(r)$ is called a slowly changing function if for $\varepsilon(>0)$,

$$
\frac{1}{k^{\varepsilon}} \leq \frac{L(k r)}{L(r)} \leq k^{\varepsilon} \text { for } r \geq r(\varepsilon) \text { and }
$$

uniformly for $k(\geq 1)$.

If further, $L(r)$ is differentiable, the above condition is equivalent to

$$
\lim _{r \rightarrow \infty} \frac{r L^{\prime}(r)}{L(r)}=0
$$

Somasundaram and Thamizharasi [8] introduced the notions of $L$-order ( $L$-lower order ) for entire functions where $L \equiv L(r)$ is a positive continuous function increasing slowly i.e., $L(a r) \sim L(r)$ as $r \rightarrow \infty$ for every positive constant ' $a$ '. The more generalised concept for $L$-order ( $L$-lower order ) for entire function are $L^{*}$-order $\left(L^{*}\right.$-lower order $)$. Their definitions are as follows:

Definition 5 [8]The $L^{*}$-order $\rho_{f}^{L^{*}}$ and the $L^{*}$-lower order $\lambda_{f}^{L^{*}}$ of an entire function $f$ are defined as

$$
\rho_{f}^{L^{*}}=\limsup _{r \rightarrow \infty} \frac{\log ^{[2]} M(r, f)}{\log \left[r e^{L(r)}\right]} \text { and } \lambda_{f}^{L^{*}}=\liminf _{r \rightarrow \infty} \frac{\log ^{[2]} M(r, f)}{\log \left[r e^{L(r)}\right]} .
$$

In the line of Sato [6], Datta and Biswas [2] one can define the generalised $L^{*}$-order $\rho_{f}^{[m] L^{*}}$ and generalised $L^{*}$-lower order $\lambda_{f}^{[m] L^{*}}$ of an entire function $f$ in the following manner :

Definition 6 Let $m$ be an integer $\geq 1$. The generalised $L^{*}$-order $\rho_{f}^{[m] L^{*}}$ and generalised $L^{*}$-lower order $\lambda_{f}^{[m] L^{*}}$ of an entire function $f$ are defined as

$$
\rho_{f}^{[m] L^{*}}=\limsup _{r \rightarrow \infty} \frac{\log ^{[m]} M(r, f)}{\log \left[r e^{L(r)}\right]} \text { and } \lambda_{f}^{[m] L^{*}}=\liminf _{r \rightarrow \infty} \frac{\log ^{[m]} M(r, f)}{\log \left[r e^{L(r)}\right]}
$$

respectively.

Datta, Biswas and Hoque [3] reformulated Definition 6 in terms of the maximum terms of entire functions in the following way: 
Definition 7 [3] The growth indicators $\rho_{f}^{[m] L^{*}}$ and $\lambda_{f}^{[m] L^{*}}$ for an entire function $f$ are defined as

$$
\rho_{f}^{[m] L^{*}}=\limsup _{r \rightarrow \infty} \frac{\log ^{[m]} \mu(r, f)}{\log \left[r e^{L(r)}\right]} \text { and } \lambda_{f}^{[m] L^{*}}=\liminf _{r \rightarrow \infty} \frac{\log ^{[m]} \mu(r, f)}{\log \left[r e^{L(r)}\right]}
$$

respectively where $m$ be an integer $\geq 1$.

Lakshminarasimhan [4] introduced the idea of the functions of $L$ bounded index. Later Lahiri and Bhattacharjee [5] worked on the entire functions of $L$-bounded index and of non uniform $L$-bounded index. In this paper we would like to investigate some growth properties of composite entire functions on the basis of their maximum terms and maximum moduli using generalised $L^{*}$-order and generalised $L^{*}$-lower order .

\section{Lemmas.}

In this section we present some lemmas which will be needed in the sequel.

Lemma 1 [9] Let $f$ and $g$ be any two entire functions with $g(0)=0$. Then for all sufficiently large values of $r$,

$$
\mu(r, f \circ g) \geq \frac{1}{2} \mu\left(\frac{1}{8} \mu\left(\frac{r}{4}, g\right)-|g(0)|, f\right) .
$$

Lemma 2 [1] If $f$ and $g$ are any two entire functions then for all sufficiently large values of $r$,

$$
M\left(\frac{1}{8} M\left(\frac{r}{2}, g\right)-|g(0)|, f\right) \leq M(r, f \circ g) \leq M(M(r, g), f) .
$$

\section{Theorems.}

In this section we present the main results of the paper.

Theorem 1 Let $f$ and $g$ be any two entire functions such that $0<\lambda_{f}^{[m] L^{*}} \leq$ $\rho_{f}^{[m] L^{*}}<\infty$ where $m \geq 1$ and $0<\lambda_{g}^{L^{*}} \leq \rho_{g}^{L^{*}}<\infty$. Then for every constant $A$ and real number $x$,

$$
\lim _{r \rightarrow \infty} \frac{\log ^{[m]} \mu(r, f \circ g)}{\left\{\log ^{[m]} \mu\left(r^{A}, f\right)\right\}^{1+x}}=\infty
$$


Proof. If $x$ is such that $1+x \leq 0$, then the theorem is obvious. So we suppose that $1+x>0$.

Now in view of Lemma 1, we get for all sufficiently large values of $r$ that

$$
\begin{array}{r}
\mu(r, f \circ g) \geq \frac{1}{2} \mu\left(\frac{1}{16} \mu\left(\frac{r}{2}, g\right), f\right) \\
i . e ., \log ^{[m]} \mu(r, f \circ g) \geq O(1)+\log ^{[m]} \mu\left(\frac{1}{16} \mu\left(\frac{r}{2}, g\right), f\right) \\
\text { i.e., } \log ^{[m]} \mu(r, f \circ g) \geq O(1)+\left(\lambda_{f}^{[m] L^{*}}-\varepsilon\right)\left[\log \left\{\frac{1}{16} \mu\left(\frac{r}{2}, g\right)\right\}\right. \\
\left.+L\left(\frac{1}{16} \mu\left(\frac{r}{2}, g\right)\right)\right] \\
\text { i.e., } \log ^{[m]} \mu(r, f \circ g) \geq O(1)+\left(\lambda_{f}^{[m] L^{*}}-\varepsilon\right)\left[\log M\left(\frac{r}{2}, g\right)+O(1)\right. \\
\left.+L\left(\frac{1}{16} \mu\left(\frac{r}{2}, g\right)\right)\right] \\
i . e ., \log ^{[m]} \mu(r, f \circ g) \geq O(1) \\
+\left(\lambda_{f}^{[m] L^{*}}-\varepsilon\right)\left\{\left(\frac{r}{2}\right) e^{L(r)}\right\}^{\lambda_{g}^{L^{*}}-\varepsilon}+O(1)+L\left(\frac{1}{16} \mu\left(\frac{r}{2}, g\right)\right)
\end{array}
$$

where we choose $0<\varepsilon<\min \left\{\lambda_{f}^{[m] L^{*}}, \lambda_{g}^{L^{*}}\right\}$.

Also for all sufficiently large values of $r$, we obtain that

$$
\begin{aligned}
\log ^{[m]} \mu\left(r^{A}, f\right) & \leq\left(\rho_{L(f)}^{[m] L^{*}}+\varepsilon\right) \log \left\{r^{A} e^{L\left(r^{A}\right)}\right\} \\
i . e ., \log ^{[m]} \mu\left(r^{A}, f\right) & \leq\left(\rho_{f}^{[m] L^{*}}+\varepsilon\right) \log \left\{r^{A} e^{L\left(r^{A}\right)}\right\} \\
\text { i.e., } \quad\left\{\log ^{[m]} \mu\left(r^{A}, f\right)\right\}^{1+x} & \leq\left(\rho_{f}^{[m] L^{*}}+\varepsilon\right)^{1+x}\left(A \log r+L\left(r^{A}\right)\right)^{1+x} .
\end{aligned}
$$

Therefore from (1) and (2) it follows for all sufficiently large values of $r$ that

$$
\begin{aligned}
& \frac{\log ^{[m]} \mu(r, f \circ g)}{\left\{\log ^{[m]} \mu\left(r^{A}, f\right)\right\}^{1+x}} \\
& \quad \geq \frac{O(1)+\left(\lambda_{f}^{[m] L^{*}}-\varepsilon\right)\left\{\left(\frac{r}{2}\right) e^{L(r)}\right\}^{\lambda_{g}^{L^{*}}-\varepsilon}+O(1)+L\left(\frac{1}{16} \mu\left(\frac{r}{2}, g\right)\right)}{\left(\rho_{f}^{[m] L^{*}}+\varepsilon\right)^{1+x}\left(A \log r+L\left(r^{A}\right)\right)^{1+x}} .
\end{aligned}
$$


Thus the theorem follows from (3).

In the line of Theorem 1, we may establish the following theorem for the right factor of the composite entire function :

Theorem 2 Let $f$ and $g$ be any two entire functions with $0<\lambda_{f}^{[m] L^{*}} \leq$ $\rho_{f}^{[m] L^{*}}<\infty$ and $0<\lambda_{g}^{L^{*}} \leq \rho_{g}^{L^{*}}<\infty$ where $m \geq 1$. Then for every constant $A$ and real number $x$,

$$
\lim _{r \rightarrow \infty} \frac{\log ^{[m]} \mu(r, f \circ g)}{\left\{\log ^{[2]} \mu\left(r^{A}, g\right)\right\}^{1+x}}=\infty
$$

The proof is omitted.

Theorem 3 Let $f$ and $g$ be any two entire functions such that $0<\lambda_{f}^{[m] L^{*}} \leq$ $\rho_{f}^{[m] L^{*}}<\infty$ and $0<\lambda_{g}^{L^{*}} \leq \rho_{g}^{L^{*}}<\infty$ where $m \geq 1$. Then for any two positive integers $\alpha$ and $\beta$,

$$
\lim _{r \rightarrow \infty} \frac{\log ^{[m+1]} \mu\left(\exp \left(\exp \left(r^{\alpha}\right)\right), f \circ g\right)}{\log ^{[m]} \mu\left(\exp \left(r^{\beta}\right), f\right)+L\left(\exp \left(\exp \left(r^{\alpha}\right)\right)\right)}=\infty,
$$

where $K(r, \alpha ; L)=\left\{\begin{array}{l}0 \text { if } r^{\beta}=o\left\{L\left(\exp \left(\exp \left(r^{\alpha}\right)\right)\right)\right\} \text { as } r \rightarrow \infty \\ L\left(\exp \left(\exp \left(r^{\alpha}\right)\right)\right) \text { otherwise }\end{array}\right.$

Proof. Taking $x=0$ and $A=1$ in Theorem 1, we obtain for $K>1$ and for all sufficiently large values of $r$ that

$$
\begin{aligned}
\log ^{[m]} \mu(r, f \circ g) & >K \log ^{[m]} \mu(r, f) \\
\text { i.e., } \log ^{[m-1]} \mu(r, f \circ g) & >\left\{\log ^{[m-1]} \mu(r, f)\right\}^{K} \\
\text { i.e., } \log ^{[m-1]} \mu(r, f \circ g) & >\left\{\log ^{[m-1]} \mu(r, f)\right\}^{K} \\
\text { i.e., } \log ^{[m-1]} \mu(r, f \circ g) & >\log ^{[m-1]} \mu(r, f)
\end{aligned}
$$

Therefore from (4) we get for all sufficiently large values of $r$ that

$$
\begin{gathered}
\log ^{[m]} \mu\left(\exp \left(\exp \left(r^{\alpha}\right)\right), f \circ g\right)>\log ^{[m]} \mu\left(\exp \left(\exp \left(r^{\alpha}\right)\right), f\right) \\
\quad \text { i.e., } \log ^{[m]} \mu\left(\exp \left(\exp \left(r^{\alpha}\right)\right), f \circ g\right) \\
>\quad\left(\lambda_{f}^{[m] L^{*}}-\varepsilon\right) \cdot \log \left\{\exp \left(\exp \left(r^{\alpha}\right)\right) \cdot \exp L\left(\exp \left(\exp \left(r^{\alpha}\right)\right)\right)\right\}
\end{gathered}
$$




$$
\begin{gathered}
\text { i.e., } \log ^{[m]} \mu\left(\exp \left(\exp \left(r^{\alpha}\right)\right), f \circ g\right) \\
>\left(\lambda_{f}^{[m] L^{*}}-\varepsilon\right) \cdot\left\{\left(\exp \left(r^{\alpha}\right)\right)+L\left(\exp \left(\exp \left(r^{\alpha}\right)\right)\right)\right\} \\
\text { i.e., } \log ^{[m]} \mu\left(\exp \left(\exp \left(r^{\alpha}\right)\right), f \circ g\right) \\
>\quad\left(\lambda_{f}^{[m] L^{*}}-\varepsilon\right) \cdot\left\{\left(\exp \left(r^{\alpha}\right)\right)\left(1+\frac{L\left(\exp \left(\exp \left(r^{\alpha}\right)\right)\right)}{\left(\exp \left(r^{\alpha}\right)\right)}\right)\right\}
\end{gathered}
$$

i.e., $\log ^{[m+1]} \mu\left(\exp \left(\exp \left(r^{\alpha}\right)\right), f \circ g\right)>O(1)+\log \exp \left(r^{\alpha}\right)$

$$
+\log \left\{1+\frac{L\left(\exp \left(\exp \left(r^{\alpha}\right)\right)\right)}{\left(\exp \left(r^{\alpha}\right)\right)}\right\}
$$

i.e., $\log ^{[m+1]} \mu\left(\exp \left(\exp \left(r^{\alpha}\right)\right), f \circ g\right)>O(1)+r^{\alpha}$

$$
+\log \left\{1+\frac{L\left(\exp \left(\exp \left(r^{\alpha}\right)\right)\right)}{\left(\exp \left(r^{\alpha}\right)\right)}\right\}
$$

i.e., $\log ^{[m+1]} \mu\left(\exp \left(\exp \left(r^{\alpha}\right)\right), f \circ g\right)>O(1)+r^{\alpha}+L\left(\exp \left(\exp \left(r^{\alpha}\right)\right)\right)$

$$
\begin{aligned}
& -\log \left[\exp \left\{L\left(\exp \left(\exp \left(r^{\alpha}\right)\right)\right)\right\}\right] \\
+ & \log \left[1+\frac{L\left(\exp \left(\exp \left(r^{\alpha}\right)\right)\right)}{\exp \left(\mu r^{\alpha}\right)}\right]
\end{aligned}
$$

i.e., $\log ^{[m+1]} \mu\left(\exp \left(\exp \left(r^{\alpha}\right)\right), f \circ g\right)>O(1)+r^{\alpha}+L\left(\exp \left(\exp \left(r^{\alpha}\right)\right)\right)$

$$
\begin{aligned}
+\log \left[\frac{1}{\exp \left\{L\left(\exp \left(\exp \left(r^{\alpha}\right)\right)\right)\right\}}\right. \\
\left.+\frac{L\left(\exp \left(\exp \left(r^{\alpha}\right)\right)\right)}{\exp \left\{L\left(\exp \left(\exp \left(r^{\alpha}\right)\right)\right)\right\} \cdot \exp \left(r^{\alpha}\right)}\right]
\end{aligned}
$$

i.e., $\quad \log ^{[m+1]} \mu\left(\exp \left(\exp \left(r^{\alpha}\right)\right), f \circ g\right)>O(1)+r^{(\alpha-\beta)} . r^{\beta}$

$$
+L\left(\exp \left(\exp \left(r^{\alpha}\right)\right)\right) \text {. }
$$

Again we have for all sufficiently large values of $r$ that

$$
\begin{aligned}
\log ^{[m]} \mu\left(\exp \left(r^{\beta}\right), f\right) & \leq\left(\rho_{f}^{[m] L^{*}}+\varepsilon\right) \log \left\{\exp \left(r^{\beta}\right) e^{L\left(\exp \left(r^{\beta}\right)\right)}\right\} \\
\text { i.e., } \log { }^{[m]} \mu\left(\exp \left(r^{\beta}\right), f\right) & \leq\left(\rho_{f}^{[m] L^{*}}+\varepsilon\right)\left\{\log \exp \left(r^{\beta}\right)+L\left(\exp \left(r^{\beta}\right)\right)\right\} \\
\text { i.e., } \quad \log ^{[m]} \mu\left(\exp \left(r^{\beta}\right), f\right) & \leq\left(\rho_{f}^{[m] L^{*}}+\varepsilon\right)\left\{r^{\beta}+L\left(\exp \left(r^{\beta}\right)\right)\right\}
\end{aligned}
$$




$$
\text { i.e., } \frac{\log ^{[m]} \mu\left(\exp \left(r^{\beta}\right), f\right)-\left(\rho_{f}^{[m] L^{*}}+\varepsilon\right) L\left(\exp \left(r^{\beta}\right)\right)}{\left(\rho_{f}^{[m] L^{*}}+\varepsilon\right)} \leq r^{\beta} .
$$

Now from (5) and (6) it follows for all sufficiently large values of $r$ that

$$
\begin{aligned}
& \log ^{[m+1]} \mu\left(\exp \left(\exp \left(r^{\alpha}\right)\right), f \circ g\right) \\
& \geq O(1)+\left(\frac{r^{(\alpha-\beta)}}{\rho_{f}^{[m] L^{*}}+\varepsilon}\right)\left[\log ^{[m]} \mu\left(\exp \left(r^{\beta}\right), f\right)-\left(\rho_{f}^{[m] L^{*}}+\varepsilon\right) L\left(\exp \left(r^{\beta}\right)\right)\right] \\
& +L\left(\exp \left(\exp \left(r^{\alpha}\right)\right)\right) \\
& \text { i.e., } \frac{\log ^{[m+1]} \mu\left(\exp \left(\exp \left(r^{\alpha}\right)\right), f \circ g\right)}{\log ^{[m]} \mu\left(\exp \left(r^{\beta}\right), f\right)} \geq \frac{L\left(\exp \left(\exp \left(r^{\alpha}\right)\right)\right)+O(1)}{\log ^{[m]} \mu\left(\exp \left(r^{\beta}\right), f\right)} \\
& +\frac{r^{(\alpha-\beta)}}{\rho_{f}^{[m] L^{*}}+\varepsilon}\left\{1-\frac{\left(\rho_{f}^{[m] L^{*}}+\varepsilon\right) L\left(\exp \left(r^{\beta}\right)\right)}{\log ^{[m]} \mu\left(\exp \left(r^{\beta}\right), f\right)}\right\} .
\end{aligned}
$$

Again from (7) we get for all sufficiently large values of $r$ that

$$
\begin{gathered}
\frac{\log ^{[m+1]} \mu\left(\exp \left(\exp \left(r^{\alpha}\right)\right), f \circ g\right)}{\log ^{[m]} \mu\left(\exp \left(r^{\beta}\right), f\right)+L\left(\exp \left(\exp \left(r^{\alpha}\right)\right)\right)} \\
\geq \frac{O(1)-r^{(\alpha-\beta)} L\left(\exp \left(r^{\beta}\right)\right)}{\log ^{[m]} \mu\left(\exp \left(r^{\beta}\right), f\right)+L\left(\exp \left(\exp \left(r^{\alpha}\right)\right)\right)} \\
+\frac{\left(\frac{r^{(\alpha-\beta)}}{\rho_{f}^{[m] L^{*}+\varepsilon}}\right) \log ^{[m]} \mu\left(\exp \left(r^{\beta}\right), f\right)}{\log ^{[m]} \mu\left(\exp \left(r^{\beta}\right), f\right)+L\left(\exp \left(\exp \left(r^{\alpha}\right)\right)\right)} \\
\quad+\frac{L\left(\exp \left(\exp \left(r^{\alpha}\right)\right)\right)}{\log ^{[m]} \mu\left(\exp \left(r^{\beta}\right), f\right)+L\left(\exp \left(\exp \left(r^{\alpha}\right)\right)\right)} \\
\text { i.e., } \frac{\frac{O(1)-r^{(\alpha-\beta)} L\left(\exp \left(r^{\beta}\right)\right)}{L\left(\exp \left(\exp \left(r^{\alpha}\right)\right)\right)}}{\log ^{[m]} \mu\left(\exp \left(r^{\beta}\right), f\right)+L\left(\exp \left(\exp \left(r^{\alpha}\right)\right)\right)} \geq \frac{\frac{\log ^{[m]} \mu\left(\exp \left(r^{\beta}\right), f\right)}{L\left(\exp \left(\exp \left(r^{\alpha}\right)\right)\right)}+1}{\left(\frac{r^{(\alpha-\beta)}}{\rho_{f}^{[m] L^{*}}+\varepsilon}\right)} \\
+\frac{L\left(\exp \left(\exp \left(r^{\alpha}\right)\right)\right)}{1+\frac{\left.\left.\exp \left(r^{\alpha}\right)\right), f \circ g\right)}{\log ^{[m]} \mu\left(\exp \left(r^{\beta}\right), f\right)}}+\frac{1+\frac{\log ^{[m]} \mu\left(\exp \left(r^{\beta}\right), f\right)}{L\left(\exp \left(\exp \left(r^{\alpha}\right)\right)\right)}}{1}
\end{gathered}
$$

Case I. If $r^{\beta}=o\left\{L\left(\exp \left(\exp \left(r^{\alpha}\right)\right)\right)\right\}$ then it follows from (8) that

$$
\liminf _{r \rightarrow \infty} \frac{\log ^{[m+1]} \mu\left(\exp \left(\exp \left(r^{\alpha}\right)\right), f \circ g\right)}{\log ^{[m]} \mu\left(\exp \left(r^{\beta}\right), f\right)}=\infty \text {. }
$$


Case II. If $r^{\beta} \neq o\left\{L\left(\exp \left(\exp \left(r^{\alpha}\right)\right)\right)\right\}$ then two sub cases may arise:

Sub case (a). If $L\left(\exp \left(\exp \left(r^{\alpha}\right)\right)\right)=o\left\{\log ^{[m]} \mu\left(\exp \left(r^{\beta}\right), f\right)\right\}$, then we get from (9) that

$$
\liminf _{r \rightarrow \infty} \frac{\log ^{[m+1]} \mu\left(\exp \left(\exp \left(r^{\alpha}\right)\right), f \circ g\right)}{\log ^{[m]} \mu\left(\exp \left(r^{\beta}\right), f\right)+L\left(\exp \left(\exp \left(r^{\alpha}\right)\right)\right)}=\infty
$$

Sub case (b). If $L\left(\exp \left(\exp \left(r^{\alpha}\right)\right)\right) \sim \log ^{[m]} \mu\left(\exp \left(r^{\beta}\right), f\right)$ then

$$
\lim _{r \rightarrow \infty} \frac{L\left(\exp \left(\exp \left(r^{\alpha}\right)\right)\right)}{\log ^{[m]} \mu\left(\exp \left(r^{\beta}\right), f\right)}=1
$$

and we obtain from (9) that

$$
\liminf _{r \rightarrow \infty} \frac{\log ^{[m+1]} \mu\left(\exp \left(\exp \left(r^{\alpha}\right)\right), f \circ g\right)}{\log ^{[m]} \mu\left(\exp \left(r^{\beta}\right), f\right)+L\left(\exp \left(\exp \left(r^{\alpha}\right)\right)\right)}=\infty .
$$

Combining Case I and Case II we obtain that

$$
\lim _{r \rightarrow \infty} \frac{\log ^{[m+1]} \mu\left(\exp \left(\exp \left(r^{\alpha}\right)\right), f \circ g\right)}{\log ^{[m]} \mu\left(\exp \left(r^{\beta}\right), f\right)+L\left(\exp \left(\exp \left(r^{\alpha}\right)\right)\right)}=\infty,
$$

where $K(r, \alpha ; L)=\left\{\begin{array}{l}0 \text { if } r^{\mu}=o\left\{L\left(\exp \left(\exp \left(r^{\alpha}\right)\right)\right)\right\} \text { as } r \rightarrow \infty \\ L\left(\exp \left(\exp \left(r^{\alpha}\right)\right)\right) \text { otherwise }\end{array}\right.$ This proves the theorem.

Theorem 4 Let $f$ and $g$ be any two entire functions with $0<\lambda_{f}^{[m] L^{*}} \leq$ $\rho_{f}^{[m] L^{*}}<\infty$ and $0<\lambda_{g}^{L^{*}} \leq \rho_{g}^{L^{*}}<\infty$ where $m \geq 1$. Then for any two positive integers $\alpha$ and $\beta$,

$$
\lim _{r \rightarrow \infty} \frac{\log ^{[m+1]} \mu\left(\exp \left(\exp \left(r^{\alpha}\right)\right), f \circ g\right)}{\log ^{[2]} \mu\left(\exp \left(r^{\beta}\right), g\right)+L\left(\exp \left(\exp \left(r^{\alpha}\right)\right)\right)}=\infty,
$$

where $K(r, \alpha ; L)=\left\{\begin{array}{l}0 \text { if } r^{\beta}=o\left\{L\left(\exp \left(\exp \left(r^{\alpha}\right)\right)\right)\right\} \text { as } r \rightarrow \infty \\ L\left(\exp \left(\exp \left(r^{\alpha}\right)\right)\right) \text { otherwise }\end{array}\right.$

The proof is omitted because it can be carried out in the line of Theorem 3 .

Remark 1 In view of Lemma 2, the results analogous to Theorem 1, Theorem 2, Theorem 3 and Theorem 4 can also be derived in terms of maximum moduli of composite entire functions. 
Theorem 5 Let $f$ and $g$ be any two entire functions such that $0<\rho_{g}^{L^{*}}<$ $\lambda_{f}^{[m] L^{*}} \leq \rho_{f}^{[m] L^{*}}<\infty$ where $m \geq 1$. Then for any $\beta>1$,

$$
\lim _{r \rightarrow \infty} \frac{\log ^{[m]} \mu(r, f \circ g)}{\log ^{[m]} \mu(r, f) \cdot K(r, g ; L)}=0
$$

where $K(r, g ; L)=\left\{\begin{array}{c}1 \text { if } L(\mu(\beta r, g))=o\left\{r^{\alpha} e^{\alpha L(r)}\right\} \text { as } r \rightarrow \infty \\ \quad \text { and for some } \alpha<\lambda_{f}^{[m] L^{*}} \\ L(\mu(\beta r, g)) \text { otherwise. }\end{array}\right.$

Proof. In view of Lemma 2 and taking $R=\beta r$ in the inequality $\mu(r, f) \leq$ $M(r, f) \leq \frac{R}{R-r} \mu(R, f)\{c f .[10]\}$, we have for all sufficiently large values of $r$ that

$$
\begin{gathered}
\mu(r, f \circ g) \leq M(r, f \circ g) \leq M(M(r, g), f) \\
\text { i.e., } \log ^{[m]} \mu(r, f \circ g) \leq \log ^{[m]} M(M(r, g), f) \\
\text { i.e., } \log ^{[m]} \mu(r, f \circ g) \leq\left(\rho_{f}^{[m] L^{*}}+\varepsilon\right)\left[\log M(r, g) e^{L(M(r, g))}\right] \\
\text { i.e., } \log ^{[m]} \mu(r, f \circ g) \leq\left(\rho_{f}^{[m] L^{*}}+\varepsilon\right)[\log M(r, g)+L(M(r, g))] \quad(10) \\
\text { i.e., } \log ^{[m]} \mu(r, f \circ g) \leq\left(\rho_{f}^{[m] L^{*}}+\varepsilon\right)\left[\left\{r e^{L(r)}\right\}^{\left(\rho_{g}^{L^{*}+\varepsilon}\right)}+L\left(\frac{\beta}{(\beta-1)} \mu(\beta r, g)\right)\right] \\
\text { i.e., } \log ^{[m]} \mu(r, f \circ g) \leq\left(\rho_{f}^{[m] L^{*}}+\varepsilon\right)\left[\left\{r e^{L(r)}\right\}^{\left(\rho_{g}^{L^{*}}+\varepsilon\right)}+L(\mu(\beta r, g))\right] . \quad(11)
\end{gathered}
$$

Also we obtain for all sufficiently large values of $r$ that

$$
\begin{aligned}
\log ^{[m]} \mu(r, f) & \geq\left(\lambda_{f}^{[m] L^{*}}-\varepsilon\right) \log \left[r e^{L(r)}\right] \\
\text { i.e., } \log ^{[m]} \mu(r, f) & \geq\left(\lambda_{f}^{[m] L^{*}}-\varepsilon\right) \log \left[r e^{L(r)}\right] \\
\text { i.e., } \log ^{[m]} \mu(r, f) & \geq\left[r e^{L(r)}\right]\left(\lambda_{f}^{[m] L^{*}}-\varepsilon\right) .
\end{aligned}
$$


Now from (11) and (12) we get for all sufficiently large values of $r$ that

$$
\frac{\log ^{[m]} \mu(r, f \circ g)}{\log ^{[m]} \mu(r, f)} \leq \frac{\left(\rho_{f}^{[m] L^{*}}+\varepsilon\right)\left[\left\{r e^{L(r)}\right\}^{\left(\rho_{g}^{L^{*}+\varepsilon}\right)}+L(\mu(\beta r, g))\right]}{\left[r e^{L(r)}\right]^{\left(\lambda_{f}^{[m] L^{*}}-\varepsilon\right)}} .
$$

Since $\rho_{g}^{L^{*}}<\lambda_{f}^{[m] L^{*}}$, we can choose $\varepsilon(>0)$ in such a way that

$$
\rho_{g}^{L^{*}}+\varepsilon<\lambda_{f}^{[m] L^{*}}-\varepsilon
$$

Case I. Let $L(\mu(\beta r, g))=o\left\{r^{\alpha} e^{\alpha L(r)}\right\}$ as $r \rightarrow \infty$ and for some $\alpha<\lambda_{f}^{[m] L^{*}}$. As $\alpha<\lambda_{f}^{[m] L^{*}}$, we can choose $\varepsilon(>0)$ in such a way that

$$
\alpha<\lambda_{f}^{[m] L^{*}}-\varepsilon .
$$

Since $L(\mu(\beta r, g))=o\left\{r^{\alpha} e^{\alpha L(r)}\right\}$ as $r \rightarrow \infty$ we get on using (15) that

$$
\begin{aligned}
& \frac{L(\mu(\beta r, g))}{r^{\alpha} e^{\alpha L(r)}} \rightarrow 0 \text { as } r \rightarrow \infty \\
& i . e ., \frac{L(\mu(\beta r, g))}{\left[r e^{L(r)]}\right]^{\left[\lambda_{f}^{[m] L^{*}}-\varepsilon\right)}} \rightarrow 0 \text { as } r \rightarrow \infty .
\end{aligned}
$$

Now in view of $(13),(14)$ and (16) we obtain that

$$
\lim _{r \rightarrow \infty} \frac{\log ^{[m]} \mu(r, f \circ g)}{\log ^{[m]} \mu(r, f)}=0 .
$$

Case II. If $L(\mu(\beta r, g)) \neq o\left\{r^{\alpha} e^{\alpha L(r)}\right\}$ as $r \rightarrow \infty$ and for some $\alpha<\lambda_{f}^{[m] L^{*}}$ then we get from (13) that for a sequence of values of $r$ tending to infinity,

$$
\begin{aligned}
& \frac{\log ^{[m]} \mu(r, f \circ g)}{\log ^{[m]} \mu(r, f) L(\mu(\beta r, g))} \leq \frac{\left(\rho_{f}^{[m] L^{*}}+\varepsilon\right)\left\{r e^{L(r)}\right\}^{\left(\rho_{g}^{L^{*}}+\varepsilon\right)}}{\left[r e ^ { L ( r ) ] } \left(^{\left[\lambda_{f}^{[m] L^{*}}-\varepsilon\right)} L(\mu(\beta r, g))\right.\right.} \\
& +\frac{\left(\rho_{f}^{[m] L^{*}}+\varepsilon\right)}{\left[r e^{L(r)]}\left(\lambda_{f}^{[m] L^{*}}-\varepsilon\right)\right.} .
\end{aligned}
$$

Now using (14) it follows from (18) that

$$
\lim _{r \rightarrow \infty} \frac{\log ^{[m]} \mu(r, f \circ g)}{\log ^{[m]} \mu(r, f) L(\mu(\beta r, g))}=0 .
$$


Combining (17) and (19) we obtain that

$$
\lim _{r \rightarrow \infty} \frac{\log ^{[m]} \mu(r, f \circ g)}{\log ^{[m]} \mu(r, f) \cdot K(r, g ; L)}=0,
$$

where $K(r, g ; L)=\left\{\begin{array}{c}1 \text { if } L(\mu(\beta r, g))=o\left\{r^{\alpha} e^{\alpha L(r)}\right\} \text { as } r \rightarrow \infty \\ \quad \text { and for some } \alpha<\lambda_{f}^{[m] L^{*}} \\ L(\mu(\beta r, g)) \text { otherwise. }\end{array}\right.$

Thus the theorem is established.

The following theorem can be carried out in the line of Theorem 5 and therefore its proof is omitted :

Theorem 6 Let $f$ and $g$ be any two entire functions with $0<\rho_{g}^{L^{*}}<\rho_{f}^{[m] L^{*}}<$ $\infty$ where $m \geq 1$. Then for any $\beta>1$,

$$
\liminf _{r \rightarrow \infty} \frac{\log ^{[m]} \mu(r, f \circ g)}{\log ^{[m]} \mu(r, f) \cdot K(r, g ; L)}=0,
$$

where $K(r, g ; L)=\left\{\begin{array}{c}1 \text { if } L(\mu(\beta r, g))=o\left\{r^{\alpha} e^{\alpha L(r)}\right\} \text { as } r \rightarrow \infty \\ \quad \text { and for some } \alpha<\rho_{f}^{[m] L^{*}} \\ L(\mu(\beta r, g)) \text { otherwise. }\end{array}\right.$

Replacing maximum term by maximum modulus in Theorem 5 and Theorem 6 we respectively get Theorem 7 and Theorem 8 and therefore their proofs are omitted.

Theorem 7 Let $f$ and $g$ be any two entire functions such that $0<\rho_{g}^{L^{*}}<$ $\lambda_{f}^{[m] L^{*}} \leq \rho_{f}^{[m] L^{*}}<\infty$ where $m \geq 1$. Then

$$
\lim _{r \rightarrow \infty} \frac{\log ^{[m]} M(r, f \circ g)}{\log ^{[m]} M(r, f) \cdot K(r, g ; L)}=0
$$

where $K(r, g ; L)=\left\{\begin{array}{c}1 \text { if } L(M(r, g))=o\left\{r^{\alpha} e^{\alpha L(r)}\right\} \text { as } r \rightarrow \infty \\ \text { and for some } \alpha<\lambda_{f}^{[m] L^{*}} \\ L(M(r, g)) \text { otherwise. }\end{array}\right.$

Theorem 8 Suppose $f$ and $g$ be any two entire functions with $0<\rho_{g}^{L^{*}}<$ $\rho_{f}^{[m] L^{*}}<\infty$ where $m \geq 1$. Then

$$
\liminf _{r \rightarrow \infty} \frac{\log ^{[m]} M(r, f \circ g)}{\log ^{[m]} M(r, f) \cdot K(r, g ; L)}=0,
$$

where $K(r, g ; L)=\left\{\begin{array}{c}1 \text { if } L(M(r, g))=o\left\{r^{\alpha} e^{\alpha L(r)}\right\} \text { as } r \rightarrow \infty \\ \quad \text { and for some } \alpha<\rho_{f}^{[m] L^{*}} \\ L(M(r, g)) \text { otherwise. }\end{array}\right.$ 
Theorem 9 Let $f$ and $g$ be any two entire functions with $\rho_{f}^{[m] L^{*}}<\infty, 0<$ $\lambda_{g}^{L^{*}} \leq \rho_{g}^{L^{*}}<\infty$ where $m$ is any positive integer. Then for any $\beta>1$,

(a) If $L(\mu(\beta r, g))=o\left\{\log ^{[2]} \mu(r, g)\right\}$ then

$$
\limsup _{r \rightarrow \infty} \frac{\log ^{[m+1]} \mu(r, f \circ g)}{\log ^{[2]} \mu(r, g)+L(\mu(\beta r, g))} \leq \frac{\rho_{g}^{L^{*}}}{\lambda_{g}^{L^{*}}}
$$

and $(b)$ if $\log ^{[2]} \mu(r, g)=o\{L(\mu(\beta r, g))\}$ then

$$
\lim _{r \rightarrow \infty} \frac{\log ^{[m+1]} \mu(r, f \circ g)}{\log ^{[2]} \mu(r, g)+L(\mu(\beta r, g))}=0 .
$$

Proof. Taking $R=\beta r$ in the inequality

$$
\mu(r, f) \leq M(r, f) \leq \frac{R}{R-r} \mu(R, f)\{c f .[10]\}
$$

and also using $\log \left\{1+\frac{O(1)+L(\mu(\beta r, g))}{\log \mu(\beta r, g)}\right\} \sim \frac{O(1)+L(\mu(\beta r, g))}{\log \mu(\beta r, g)}$, for all sufficiently large values of $r$ we obtain from (10) that

$$
\begin{array}{r}
\log ^{[m]} \mu(r, f \circ g) \\
\leq\left(\rho_{f}^{[m] L^{*}}+\varepsilon\right)\left[\log \mu(\beta r, g)+O(1)+L\left(\frac{\beta}{(\beta-1)} \mu(\beta r, g)\right)\right] \\
\text { i.e., } \log ^{[m]} \mu(r, f \circ g) \leq\left(\rho_{f}^{[m] L^{*}}+\varepsilon\right) \log \mu(\beta r, g)\left[1+\frac{O(1)+L(\mu(\beta r, g))}{\log \mu(\beta r, g)}\right] \\
\text { i.e., } \log ^{[m+1]} \mu(r, f \circ g) \leq \log \left(\rho_{f}^{[m] L^{*}}+\varepsilon\right)+\log \left\{1+\frac{O(1)+L(\mu(\beta r, g))}{\log \mu(\beta r, g)}\right\} \\
\text { i.e., } \log ^{[m+1]} \mu(r, f \circ g) \leq \log \left(\rho_{f}^{[m] L^{*}}+\varepsilon\right)+\left(\rho_{g}^{L^{*}}+\varepsilon\right) \log \left\{\beta r e^{L(\beta r)}\right\} \\
+\log \left\{1+\frac{O(1)+L(\mu(\beta r, g))}{\log \mu(\beta r, g)}\right\} \\
\text { i.e., } \log ^{[m+1]} \mu(r, f \circ g) \leq \log \left(\rho_{f}^{[m] L^{*}}+\varepsilon\right)+\left(\rho_{g}^{L^{*}}+\varepsilon\right) \log \left\{\beta r e^{L(r)}\right\} \\
+\log \left\{1+\frac{O(1)+L(\mu(\beta r, g))}{\log \mu(\beta r, g)}\right\}
\end{array}
$$




$$
\begin{gathered}
\text { i.e., } \log { }^{[m+1]} \mu(r, f \circ g) \leq O(1)+\left(\rho_{g}^{L^{*}}+\varepsilon\right)\{\log \beta r+L(r)\}+\frac{O(1)+L(\mu(\beta r, g))}{\log \mu(\beta r, g)} \\
\text { i.e., } \log ^{[m+1]} \mu(r, f \circ g) \leq O(1)+\left(\rho_{g}^{L^{*}}+\varepsilon\right)\{\log r+L(r)\} \\
+\left(\rho_{g}^{L^{*}}+\varepsilon\right) \log \beta+\frac{O(1)+L(\mu(\beta r, g))}{\log \mu(\beta r, g)} .
\end{gathered}
$$

Again from the definition of $L^{*}$-lower order, we get for all sufficiently large values of $r$ that

$$
\begin{aligned}
\log ^{[2]} \mu(r, g) & \geq\left(\lambda_{g}^{L^{*}}-\varepsilon\right) \log \left[r e^{L(r)}\right] \\
\text { i.e., } \quad \log ^{[2]} \mu(r, g) & \geq\left(\lambda_{g}^{L^{*}}-\varepsilon\right) \log \left[r e^{L(r)}\right] \\
\text { i.e., } \quad \log { }^{[2]} \mu(r, g) & \geq\left(\lambda_{g}^{L^{*}}-\varepsilon\right)[\log r+L(r)] \\
\text { i.e., } \log r+L(r) & \leq \frac{\log ^{[2]} \mu(r, g)}{\left(\lambda_{g}^{L^{*}}-\varepsilon\right)} .
\end{aligned}
$$

Hence from (20) and (21) it follows for all sufficiently large values of $r$ that

$$
\begin{aligned}
& \log ^{[m+1]} \mu(r, f \circ g) \\
& \leq O(1)+\left(\frac{\rho_{g}^{L^{*}}+\varepsilon}{\lambda_{g}^{L^{*}}-\varepsilon}\right) \cdot \log ^{[2]} \mu(r, g)+\left(\rho_{g}^{L^{*}}+\varepsilon\right) \log \beta+\frac{O(1)+L(\mu(\beta r, g))}{\log \mu(\beta r, g)} \\
& \text { i.e, } \frac{\log ^{[m+1]} \mu(r, f \circ g)}{\log ^{[2]} \mu(r, g)+L(\mu(\beta r, g))} \\
& \leq \frac{O(1)+\left(\rho_{g}^{L^{*}}+\varepsilon\right) \log \beta}{\log ^{[2]} \mu(r, g)+L(\mu(\beta r, g))}+\left(\frac{\rho_{g}^{L^{*}}+\varepsilon}{\lambda_{g}^{L^{*}}-\varepsilon}\right) \cdot \frac{\log ^{[2]} \mu(r, g)}{\log ^{[2]} \mu(r, g)+L(\mu(\beta r, g))} \\
& +\frac{O(1)+L(\mu(\beta r, g))}{\left[\log ^{[2]} \mu(r, g)+L(\mu(\beta r, g))\right] \log \mu(\beta r, g)} \\
& i . e, \frac{\log ^{[m+1]} \mu(r, f \circ g)}{\log ^{[2]} \mu(r, g)+L(\mu(\beta r, g))} \leq \frac{\frac{O(1)+\left(\rho_{g}^{L^{*}}+\varepsilon\right) \log \beta}{L(\mu(\beta r, g))}}{\frac{\log ^{[2]} \mu(r, g)}{L(\mu(\beta r, g))}+1}+\frac{\left(\frac{\rho_{g}^{L^{*}}+\varepsilon}{\lambda_{g}^{L *}-\varepsilon}\right)}{1+\frac{L(\mu(\beta r, g))}{\log ^{[2]} \mu(r, g)}} \\
& +\frac{1}{\left[1+\frac{\log ^{[2]} \mu(r, g)}{L(\mu(\beta r, g))}\right] \log \mu(\beta r, g)} .
\end{aligned}
$$


Since $L(\mu(\beta r, g))=o\left\{\log ^{[2]} \mu(r, g)\right\}$ as $r \rightarrow \infty$ and $\varepsilon(>0)$ is arbitrary, we obtain from (22) that

$$
\limsup _{r \rightarrow \infty} \frac{\log ^{[m+1]} \mu(r, f \circ g)}{\log ^{[2]} \mu(r, g)+L(\mu(\beta r, g))} \leq \frac{\rho_{g}^{L^{*}}}{\lambda_{g}^{L^{*}}} .
$$

Again if $\log ^{[2]} \mu(r, g)=o\{L(\mu(\beta r, g))\}$ then from (22) we get that

$$
\lim _{r \rightarrow \infty} \frac{\log ^{[m+1]} \mu(r, f \circ g)}{\log ^{[2]} \mu(r, g)+L(\mu(\beta r, g))}=0 .
$$

Thus the theorem follows from (23) and (24).

Corollary 1 Let $f$ and $g$ be any two entire functions with $\rho_{f}^{[m] L^{*}}<\infty$ and $0<\rho_{g}^{L^{*}}<\infty$ where $m \geq 1$. Then for any $\beta>1$,

(a) if $L(\mu(\beta r, g))=o\left\{\log ^{[2]} \mu(r, g)\right\}$ then

$$
\liminf _{r \rightarrow \infty} \frac{\log ^{[m+1]} \mu(r, f \circ g)}{\log ^{[2]} \mu(r, g)+L(\mu(\beta r, g))} \leq 1
$$

and $(b)$ if $\log ^{[2]} \mu(r, g)=o\{L(\mu(\beta r, g))\}$ then

$$
\liminf _{r \rightarrow \infty} \frac{\log ^{[m+1]} \mu(r, f \circ g)}{\log ^{[2]} \mu(r, g)+L(\mu(\beta r, g))}=0 .
$$

We omit the proof of Corollary 1 because it can be carried out in the line of Theorem 7 .

Remark 2 The equality sign in Theorem 5 and Corollary 1 cannot be removed as we see in the following example:

Example 1 Let $f=g=\exp z, m=2, \beta=2$ and $L(r)=\frac{1}{p} \exp \left(\frac{1}{r}\right)$ where $p$ is any positive real number.

Then

$$
\rho_{f}^{L^{*}}=\lambda_{g}^{L^{*}}=\rho_{g}^{L^{*}}=1
$$

Now

$$
\begin{aligned}
& \log \mu(r, f \circ g) \leq \log M(r, f \circ g)=\exp r \\
& \text { and } 2 \mu(2 r, g) \geq M(r, g)=\exp r
\end{aligned}
$$


Also

$$
\begin{aligned}
\log \mu(r, f \circ g) & \geq \log M\left(\frac{r}{2}, f \circ g\right)+O(1)=\exp \left(\frac{r}{2}\right)+O(1), \\
\text { and } \mu(r, g) & \leq M(r, g)=\exp r
\end{aligned}
$$

So

$$
L(M(r, g))=L(\exp r)=\frac{1}{p} \exp \left(\frac{1}{\exp r}\right)
$$

Hence

$$
\limsup _{r \rightarrow \infty} \frac{\log ^{[3]} \mu(r, f \circ g)}{\log ^{[2]} \mu(r, g)+L(\mu(\beta r, g))} \leq \limsup _{r \rightarrow \infty} \frac{\log r}{\log r+O(1)+\frac{1}{p} \exp \left(\frac{1}{\exp r}\right)}=1
$$

and

$$
\liminf _{r \rightarrow \infty} \frac{\log ^{[3]} \mu(r, f \circ g)}{\log ^{[2]} \mu(r, g)+L(\mu(\beta r, g))} \geq \liminf _{r \rightarrow \infty} \frac{\log r+O(1)}{\log r+\frac{1}{p} \exp \left(\frac{1}{\exp r}\right)}=1 .
$$

Therefore

$\liminf _{r \rightarrow \infty} \frac{\log ^{[3]} \mu(r, f \circ g)}{\log ^{[2]} \mu(r, g)+L(\mu(\beta r, g))}=\limsup _{r \rightarrow \infty} \frac{\log ^{[3]} \mu(r, f \circ g)}{\log ^{[2]} \mu(r, g)+L(\mu(\beta r, g))}=1$.

Theorem 10 Let $f$ and $g$ be any two entire functions with $\rho_{f}^{[m] L^{*}}<\infty$ and $0<\lambda_{g}^{L^{*}} \leq \rho_{g}^{L^{*}}<\infty$ where $m$ is any positive integer. Then

(a) if $L(M(r, g))=o\left\{\log ^{[2]} M(r, g\}\right.$ then

$$
\limsup _{r \rightarrow \infty} \frac{\log ^{[m+1]} M(r, f \circ g)}{\log ^{[2]} M(r, g)+L(M(r, g))} \leq \frac{\rho_{g}^{L^{*}}}{\lambda_{g}^{L^{*}}}
$$

and (b) if $\log ^{[2]} M(r, g)=o\{L(M(r, g))\}$ then

$$
\lim _{r \rightarrow \infty} \frac{\log ^{[m+1]} M(r, f \circ g)}{\log ^{[2]} M(r, g)+L(M(r, g))}=0 .
$$

Corollary 2 Let $f$ and $g$ be any two entire functions with $\rho_{f}^{[m] L^{*}}<\infty$ and $0<\rho_{g}^{L^{*}}<\infty$ where $m \geq 1$. Then for any $\beta>1$,

(a) if $L(M(r, g))=o\left\{\log ^{[2]} M(r, g)\right\}$ then

$$
\liminf _{r \rightarrow \infty} \frac{\log ^{[m+1]} M(r, f \circ g)}{\log ^{[2]} M(r, g)+L(M(r, g))} \leq 1
$$


and $(b)$ if $\log ^{[2]} M(r, g)=o\{L(M(r, g))\}$ then

$$
\liminf _{r \rightarrow \infty} \frac{\log ^{[m+1]} M(r, f \circ g)}{\log ^{[2]} M(r, g)+L(M(r, g))}=0 .
$$

We omit the proof of Theorem 10 and Corollary 2 because in view of Lemma 2 it can be carried out in the line of Theorem 9 and Corollary 1 respectively.

Remark 3 Considering $f=g=\exp z, m=2$ and $L(r)=\frac{1}{p} \exp \left(\frac{1}{r}\right)$ where $p$ is any positive real number, one can easily verify that the equality sign in Theorem 10 and Corollary 2 cannot be removed.

\section{Acknowledgement}

The authors are thankful to Professor Huzoor H. Khan, Department of Mathematics, Aligarh Muslim University, Aligarh- 202002 (U.P.), India for his Constant encouragement to carry out the paper.

\section{References}

[1] J. Clunie : The composition of entire and meromorphic functions, Mathematical Essays dedicated to A. J. Macintyre,Ohio University Press (1970), pp. 75-92.

[2] S.K. Datta and T. Biswas : On the definition of a meromorphic function of order zero, International Mathematical Forum, Vol.4, No. 37(2009) pp.1851-1861.

[3] S. K. Datta, T. Biswas and Md. A. Hoque : Maxumum modulus and maxumum term based growth analysis of entire function in the light of slowly changing function, Investigations in Mathematical Sciences, Vol. 3, No. 1 (2013), pp. 113-125.

[4] T.V. Lakshminarasimhan : A note on entire functions of bounded index, J. Indian Math. Soc., Vol. 38 (1974), pp. 43-49.

[5] I. Lahiri and N.R. Bhattacharjee : Functions of L-bounded index and of non-uniform L-bounded index, Indian J. Math., Vol. 40 (1998), No. 1, pp. 43-57.

[6] D. Sato : On the rate of growth of entire functions of fast growth, Bull. Amer. Math. Soc., Vol. 69 (1963), pp.411-414.

[7] S.K. Singh and G.P. Barker : Slowly changing functions and their applications, Indian J. Math., Vol. 19 (1977), No. 1, pp 1-6. 
[8] D. Somasundaram and R. Thamizharasi : A note on the entire functions of L-bounded index and L-type, Indian J. Pure Appl. Math., Vol.19(March 1988), No. 3, pp. 284-293.

[9] A. P. Singh : On maximum term of composition of entire functions, Proc. Nat. Acad. Sci. India, Vol. 59(A), Part I (1989), pp. 103-115.

[10] A. P. Singh and M. S. Baloria : On maximum modulus and maximum term of composition of entire functions, Indian J. Pure Appl. Math., Vol. 22, No 12(1991), pp. 1019-1026.

[11] G. Valiron : Lectures on the general theory of integral functions, Chelsea Publishing Company, 1949.

Received: July 1, 2013 